Cahiers de philosophie de l'université de

\title{
Hannah Arendt. Le moment politique de l'Europe
}

Étienne Tassin

\section{(2) OpenEdition}

Journals

Édition électronique

URL : https://journals.openedition.org/cpuc/1171

DOI : 10.4000/cpuc. 1171

ISSN : 2677-6529

Éditeur

Presses universitaires de Caen

Édition imprimée

Date de publication : 15 décembre 2010

Pagination : 165-180

ISBN : 978-2-84133-365-3

ISSN : 1282-6545

Référence électronique

Étienne Tassin, « Hannah Arendt. Le moment politique de l'Europe», Cahiers de philosophie de l'université de Caen [En ligne], 47 | 2010, mis en ligne le 02 septembre 2020, consulté le 02 février 2023. URL : http://journals.openedition.org/cpuc/1171; DOI : https://doi.org/10.4000/cpuc.1171

Creative Commons - Attribution - Pas d'Utilisation Commerciale 4.0 International - CC BY-NC 4.0 https://creativecommons.org/licenses/by-nc/4.0/ 


\section{Hannah Arendt. Le moment politique de l'Europe}

\section{L'Europe dans la brèche: rupture et négation de la tradition}

Hannah Arendt s'est efforcée de penser la brèche temporelle qui, avec les deux guerres mondiales et le totalitarisme européen, a séparé la vieille Europe de celle à venir. Comme elle l'écrit dans Between Past and Futur, le fil de la tradition est maintenant rompu ${ }^{1}$. Cette rupture du fil qui relie l'Europe d'après-guerre à sa provenance grecque, romaine et hébraïque s'est manifestée historiquement dès le début de ce siècle ${ }^{2}$ : la Grande Guerre en fut, au moins, l'expression. Du point de vue de la philosophie politique, on peut avancer que la tradition de pensée née avec Platon et Aristote a, elle, pris fin avec Karl Marx ${ }^{3}$. Ces deux aspects de la rupture, historique et philosophique, sont distincts et décalés, histoire politique et philosophie ne relevant pas de la même chronicité.

La disparition de la tradition dans le monde moderne signifie cependant que celle-ci n'organise plus notre compréhension et notre usage du monde. Elle n'implique pas un oubli du passé, au contraire: «il se pourrait qu'aujourd'hui seulement le passé s'ouvrît à nous avec une fraîcheur inattendue et nous dît des choses pour lesquelles personne encore n'a eu d'oreilles ${ }^{4}$. Mais nul philosophe rigoureux ne saurait négliger l'avertissement que cette rupture fait résonner: «nous sommes en danger d'oubli ${ }^{5}$. S'il s'agit de penser l'Europe d'aujourd'hui, il nous faut le faire depuis

1. H. Arendt, La crise de la culture [Between Past and Futur], trad. fr. sous la direction de P. Lévy, Paris, Gallimard, 1972 (dorénavant cité CC). La préface thématise cette rupture de la tradition dans les termes d'une brèche temporelle, les chapitres qui suivent en analysent les effets sur nos catégories de pensée.

2. H. Arendt, "Qu'est-ce que l'autorité?», CC, p. 121.

3. H. Arendt, «Tradition et âge moderne», CC, p. 28.

4. H. Arendt, "Qu'est-ce que l'autorité?», p. 125.

5. Ibid., p. 125 . 
cet entre-deux, depuis cette brèche temporelle qui rompt le continuum historique, et au sein de laquelle se déploie notre présent entre un passé en danger d'oubli et un avenir incertain, inenvisageable dès lors qu'il est privé des orientations que la tradition s'employait jusqu'alors à esquisser pour lui. L'Europe doit être approchée depuis ce non-temps et ce non-lieu dans lequel la pensée fait l'épreuve du désarroi puisque la tradition n'est plus décisive, mais depuis lequel l'occasion lui est offerte de penser à nouveau, et de penser le nouveau. À supposer bien sûr qu'elle sache s'affranchir des anciennes catégories, qu'elle sache s'étonner à nouveau comme au premier jour devant la nouvelle donne, terrifiante, qu'offre le siècle; et qu'elle sache laisser s'inventer une nouvelle entente de son présent.

La rupture du fil de la tradition est aussi indissociable d'une entreprise de négation radicale du monde et du sens, dont l'Europe du XX fut le théâtre, et qu'on doit comprendre comme une négation de cette même tradition: "la négation radicale de toute tradition fut d'emblée le trait principal du nazisme ${ }^{6}$. Si la rupture laisse ouverte une entente neuve du passé, la négation de la tradition détruit, elle, toutes les catégories de pensée qui se sont forgées en son sein; elle les invalide, pourrait-on dire, ou du moins jette-t-elle sur elles le soupçon qu'elles ne sauraient être opérantes pour penser le nouveau. Comment penser aujourd'hui l'Europe qui a donné naissance au totalitarisme à l'aide des catégories de pensée forgées au cours de cette tradition européenne niée par ce totalitarisme qu'elle a cependant laissé se déployer en son sein? La difficulté indique à la fois que la tradition européenne est liée à l'avènement du totalitarisme - comment le nieraiton? - mais aussi que la négation de la tradition opérée par le nazisme européen invalide les repères du jugement, les concepts et les principes que celle-ci avait élaborés pour penser ${ }^{7}$. Il nous faut donc penser l'Europe sans le recours de la tradition en pensant en elle ce qui a conduit à sa propre négation dans la négation de celle-là. Et il nous revient aussi de penser l'avenir de l'Europe dans un monde nouveau en inventant les manières de comprendre ce qui est en train d'advenir et pour lequel nous sommes privés de guide: «le passé n'éclairant plus l'avenir, l'esprit marche dans les ténèbres $»^{8}$.

6. H. Arendt, "Approches du "problème allemand" » [1945], Penser l'événement, trad. fr. sous la direction de C. Habib, Paris, Belin, 1989, p. 38.

7. Sans qu'il soit pour autant nécessaire de compromettre la tradition philosophique dans l'avènement de l'extermination: «Que nous aimions Thomas d'Aquin, Machiavel, Luther, Kant, Hegel ou Nietzsche [...] ils ne sont en rien responsables de ce qui s'est passé dans les camps d'extermination. Idéologiquement, le nazisme n'a à ses débuts aucun fondement dans la tradition...» (ibid., p. 37).

8. La formule d'A. de Tocqueville (De la démocratie en Amérique, Paris, Gallimard, 1951, t. 2, chap. VIII, p. 336) est citée par Hannah Arendt dans CC, préface, p. 15. 
Rupture et négation de la tradition marquent en effet l'avènement d'un monde nouveau, le nôtre, dont Hannah Arendt a pu dire par ailleurs qu'il naquit à Hiroshima le 6 août $1945^{\circ}$. Ce monde nouveau, monde de la production des forces destructrices du monde, est le legs d'un monde perdu: il ne saurait être pensé à l'image de ce dernier. Au regard de cette rupture d'avec la tradition qui caractérise l'Europe au lendemain de la Première Guerre mondiale, héritiers de sa négation opérée par le nazisme au cours de la Seconde Guerre, contemporains d'un monde dont la naissance est annoncée par l'explosion de la bombe atomique et qui déjà se divise à Yalta en deux camps irréconciliables, nous devons convenir, selon les mots de René Char qu'Arendt a si souvent rappelés, que «notre héritage n'est précédé d'aucun testament ${ }^{10}$. Une Europe intestat est morte au mitan du siècle: à quoi ressemblera celle qui naîtra de cette catastrophe? Et comment prétendrions-nous, nous philosophes, en saisir et le sens et la carrière à venir? On ne saurait désormais réactiver l'ancienne entente de l'Europe et faire comme si rien n'avait eu lieu ni pour le monde ni pour la pensée. Aussi est-il impossible, et vain, de prétendre réactiver au sortir du cataclysme européen, et dans le contexte naissant de l'équilibre nucléaire, une entente de l'Europe qui, si elle pouvait encore être défendue à la veille de la guerre, sonne en son lendemain comme une illusion perdue: celle que proposait, avec fermeté et conviction, le diagnostic husserlien de la Krisis, tout habité - non sans tonalité tragique - d'une irréfragable confiance en la raison.

\section{Le désaveu de l'Europe philosophique}

La figure spirituelle de l'Europe que reconstituait Husserl dans la conférence de Vienne en 1935 était supposée révéler l'entéléchie d'une humanité européenne qui, depuis l'Urphänomen philosophique auquel correspond sa naissance grecque, est vouée à l'universalité de la theoria. Animée d'un intérêt purement théorétique - «absolument non pratique» précisait Husserl - qui fait du philosophe le fonctionnaire de l'humanité, la philosophie a donné naissance, sous le nom d'Europe, à une humanité supranationale. Aussi l'Europe spirituelle ne désigne-t-elle pas dans son principe la juxtaposition de nations différentes mais ce nouvel esprit susceptible d'exercer

9. H. Arendt, Condition de l'homme moderne, G. Fradier (trad. fr.), Paris, Calmann-Lévy, 1981, p. 13. Sur le rôle de la bombe atomique sur l'idée européenne, voir «Europe and the Atom Bomb", Essays in Understanding, 1930-1954, J. Kohn (éd.), New York - San Diego - Londres, Harcourt Brace, 1994, p. 418 sq.

10. R. Char, «Feuillets d'Hypnos» [1943-1944], Fureur et mystère, Paris, Gallimard (Poésie; 15), $1967, n^{\circ} 62$, p. 102. Cité par Arendt dans $C C$, préface, p. 11, 14. 
une «fonction rectrice à l'égard de l'humanité tout entière» en la réglant sur les principes de l'universalité absolue, de l'infinité du savoir et du tout de la vérité. La crise de l'humanité européenne pouvait alors être analysée comme l'affaissement de cet esprit philosophique universel dans les volontés nationales sous l'effet d'un dévoiement de la raison en un rationalisme objectiviste et naturaliste. Car la parcellisation de l'esprit qui en résulte élève l'esprit contre lui-même; et les nations contre l'Europe. Voilà ce qu'il advenait, pour Husserl, de l'Europe en ce premier tiers du XX' ${ }^{\mathrm{e}}$ siècle. L'Europe altérée dans son essence spirituelle ne renaîtra à son destin philosophique, que le rationalisme moderne a dénaturé, qu'à condition d'un héroïsme de la raison porté par la foi en l'immortalité de l'esprit ${ }^{11}$.

On est en droit de penser que la foi en la raison a été impuissante face au déchaînement systématisé d'un rationalisme de la domination et donc de l'extermination. Et l'on doit aussi reconnaître que cette foi en la raison aura pu nourrir un aveuglement sur l'œuvre de la rationalité européenne, qui masque ce qu'il nous faut, après-coup, reconnaître: à savoir que le naturalisme et l'objectivisme qu'Husserl dénonce comme une déviation ou un détournement de la raison auront été en réalité son seul accomplissement historique. Sans s'y réduire, bien sûr, «Europe» est aussi le nom de cet accomplissement destructeur qu'Arendt a tenté de comprendre en forgeant une catégorie de pensée nouvelle, celle de totalitarisme, vouée à rendre compte d'un phénomène jusqu'alors inédit qui est à la fois le produit et la fin de la modernité européenne, accomplissement et négation de la tradition.

Aussi, dix ans après la conférence de Vienne, la représentation de l'Europe comme continent de l'esprit et de la raison voué à un destin d'universalité peut paraître simple folie, ou pure illusion. Car on ne saurait penser philosophiquement l'Europe en 1945, et après, comme on croyait encore pouvoir le faire en 1935. À cette dernière date, en raison de la foi en cette adéquation de l'Europe et de la raison philosophique, il n'était pas encore impossible à Husserl d'affirmer la suprématie des idées sur les forces empiriques et de compter sur la force des idées pour combattre l'effondrement de l'esprit dont le nazisme se révélait l'expression. En septembre 1946, Jean Lescure, parmi d'autres qui font le même constat, déclare aux premières Rencontres internationales de Genève consacrées à L'esprit européen que «le moment historique est déjà passé où l'Europe pouvait se présenter comme un problème philosophique. Sa mise en

11. E. Husserl, «La crise de l'humanité européenne et la philosophie» [mai 1935], La crise des sciences européennes et la phénoménologie transcendantale, G. Granel (trad.), Paris, Gallimard, 1976, p. 347-383. 
question serait donc aujourd'hui de nature politique». Et d'ajouter: «Je dirai que l'Europe ne peut plus se situer sur le plan de l'esprit, mais sur le plan des forces $»^{12}$.

Il y a différentes manières de situer l'Europe sur le plan des forces. La plus commune est de la considérer sous l'angle géopolitique. En 1946, on peut suggérer que l'enjeu est moins l'esprit européen que la manière dont l'Europe pourra résister et s'opposer aux deux puissances rivales qui se partagent le monde et qu'on nomme alors l'américanisme et le soviétisme. Les forces en jeu sont ici surtout économiques et militaires. La manière arendtienne d'assumer ce passage du plan philosophique au plan des forces consiste, elle, à rapporter l'intelligence de l'Europe à sa dimension et à sa signification politiques. Cette compréhension de l'Europe, comme réalité politique à venir et non plus comme entité spirituelle héritée, est corrélative d'une critique des blocages constitutifs de la philosophie politique classique, mais aussi de la phénoménologie. Husserl croyait pouvoir, entre 1935 et 1938, invoquer contre la montée du nazisme un suprême retour de l'Europe à son destin philosophique initial. Arendt laisse entendre après guerre que cette foi en la raison est un symptôme de l'aveuglement philosophique envers la politique, aveuglement consubstantiel à la tradition européenne de la philosophie politique ${ }^{13}$. Là où le premier mobilise une entente philosophique de l'Europe qui tente de la faire coïncider avec la philosophie, la raison et l'universalité de la vérité, la seconde invoque une aventure historique à venir, nourrie de la résistance au fascisme, qui doit réunir les États et les peuples européens en une organisation politique commune susceptible de prévenir le retour du totalitarisme. De l'un à l'autre est non seulement symbolisé le passage d'une Europe philosophique à une Europe politique, mais aussi le passage concomitant d'une philosophie en extériorité et en surplomb du politique - et qui pense pouvoir déterminer le «concept d'Europe en tant que téléologie historique de buts rationnels infinis» ${ }^{14}$-, à une expérience historique qui réclame dans ses errements et ses tâtonnements

12. Cf. L'esprit européen, Paris, O. Zeluck, 1947, p. 119. L’ouvrage réunit le texte des conférences et des entretiens des premières Rencontres internationales de Genève consacrées à ce thème (septembre 1946). Il est dorénavant consultable en ligne à l'adresse suivante: http://www. rencontres-int-geneve.ch/volumes_pdf/rigo1.pdf. Sur l'analyse de ces Rencontres et la signification du passage d'une pensée de l'Europe d'un plan philosophique à un plan proprement politique, je me permets de renvoyer à É. Tassin, «De l'Europe philosophique à l'Europe politique", in Existe-t-il une Europe philosophique?, N. Weil (éd.), Rennes, Presses universitaires de Rennes, 2005.

13. Cf. H. Arendt, "L'intérêt pour la politique dans la pensée philosophique européenne récente» [1954], J. Roman, A. Scala et É. Tassin (trad. fr.), Les cahiers de philosophie, ${ }^{\circ}{ }^{4}$, automne 1987, Hannah Arendt, confrontations, p. 7-28.

14. E. Husserl, «La crise de l'humanité... », p. 382 (souligné dans le texte). 
l'élucidation philosophique de la politique qui s'y joue - et qui, loin de toute spiritualité, somme l'intelligence d'assumer la dimension politique du penser philosophique.

Ce rapport de reprise critique et décalée qu'Arendt entretient avec la philosophie et la phénoménologie la conduit, de manière cohérente, à prendre au lendemain de la Seconde Guerre mondiale ses distances avec une conception philosophique de l'Europe. L'Europe que Husserl tente de sauver en démarquant les dérives naturalistes et objectivistes de la science de l'héritage platonicien, marqué par la foi en la raison et la mission rectrice de la vérité qui voueraient l'Europe à l'universalité d'un humanisme supranational, est une construction théorétique qui témoigne, dans la perspective arendtienne, de l'incapacité, structurelle si l'on peut dire, de la tradition philosophique à se plier aux événements et à accepter que la politique ne se déduise pas du concept. Au milieu du XX $X^{\mathrm{e}}$ siècle, l'Europe historique appelle une tout autre compréhension de l'Europe.

\section{Autre Europe, autre philosophie}

Comme Arendt l'a suggéré à plusieurs reprises, l'articulation de la philosophie, de la rationalité et de la quête de la vérité à «l'humanité européenne», propre à la foi en une Europe spirituelle, est l'expression par excellence d'une totale méprise sur les affaires humaines - et d'une condescendance certaine envers celles-ci -, toujours supposées soumises à la donation de sens que la raison philosophique est la seule à promettre et à réaliser ${ }^{15}$. À ses yeux le théorétisme désintéressé dont se soutient la mission d'universalité du philosopher contribue à cet aveuglement des philosophes à l'égard des situations politiques concrètes, empiriques et intéressées, comme le fut la montée du nazisme pour cette génération. On connaît le jugement qu'elle porte sur les intellectuels, singulièrement les philosophes, qui au début des années 1930 en Allemagne ne perçurent pas le sens du nazisme ou s'y laissèrent compromettre, Martin Heidegger en premier lieu ${ }^{16}$. À rebours, seul un intérêt pratique, non théorétique, fondamentalement intéressé à ce qui inter est, c'est-à-dire au monde humain concret dans son déploiement aléatoire et différencié, peut donner naissance et sens à une philosophie soucieuse des choses politiques et avertie de leurs enjeux. Certes, Arendt ne s'exprime pas expressément sur le diagnostic husserlien de la Krisis qu'elle

15. Cf. par exemple, H. Arendt, "Seule demeure la langue maternelle», entretien avec Gaus de 1964, La tradition cachée, S. Courtine-Denamy (trad. fr.), Paris, C. Bourgois, 1987, p. 221-256.

16. Cf. ibid., p. 237-238. 
n'évoque pas. Mais le jugement qu'elle porte sur la philosophie politique traditionnelle et l'effet d'aveuglement que la posture philosophique induit sur les choses politiques ne saurait laisser Husserl à part - pas plus que Heidegger, même si leurs deux pensées sont en ce point comme en d'autres divergentes, voire opposées ${ }^{17}$.

Il nous faut comprendre ce qu'elle dira à son tour de l'Europe à l'aune de cette critique conjointe de la philosophie politique et de la phénoménologie, incapables l'une et l'autre d'honorer l'étonnement requis devant les choses politiques avec autant de radicalité qu'elles sont supposées le faire pour les affaires de l'esprit. Je ne reviens pas sur cette critique de la posture traditionnelle de la philosophie devant les affaires humaines en général et les affaires politiques en particulier, Miguel Abensour en a fait une analyse judicieuse et convaincante ${ }^{18}$. On peut concevoir le projet, entrepris dès 1941-1942, d'élucider la nouvelle forme de domination totale qui s'est abattue sur l'Europe comme une réponse politique appropriée, et délibérément en rupture avec l'héritage philosophique, à la question que pose l'Europe soumise au nazisme. On ne saurait cependant restituer les arguments arendtiens en faveur d'une Europe politique, et comme on va le voir politiquement fédérale, sans mesurer à sa juste valeur l'écart que cette intelligibilité de l'Europe creuse avec ses représentations philosophique ou culturelle. Et cela afin de saisir ce qu'exige pour la pensée d'avoir à penser depuis la brèche où la plongent la rupture de la tradition et sa négation totalitaire. Car là encore, seul un rapport critique à la philosophie politique rend possible l'élucidation du moment politique de l'Europe. L'orientation fédéraliste qu'Arendt conçoit et souhaite pour l'Europe d'après-guerre ne relève pas de la "science politique » : elle a une signification philosophique indissociable d'une reformulation post-traditionnelle de ce que pourrait être une philosophie politique critique. Peut-être indique-t-elle par ailleurs, à sa façon, dans une perspective qui lui est propre, ce que Jan Patočka tentera plus tard de décrire comme la post-Europe.

Le concept philosophique d'Europe que développe Husserl, et que tentera de reformuler à sa manière Patočka dans les années 1970, est distinct du concept culturel ou du concept politique qu'on peut en produire

17. Sur le rapport de Arendt à la phénoménologie husserlienne et heideggérienne, voir É. Tassin, Le trésor perdu. Hannah Arendt, l'intelligence de l'action politique, Paris, Payot (Critique de la politique; 199), chap. II, p. 79-129; sur le rapport Arendt / Heidegger, voir D. R. Villa, Arendt et Heidegger. Le destin du politique, C. David et D. Munnich (trad. fr.), Paris, Payot (Critique de la politique), 2008.

18. M. Abensour, Hannah Arendt contre la philosophie politique?, Paris, Sens \& Tonka, 2006. 
par ailleurs ${ }^{19}$. Le concept philosophique assigne à l'Europe une naissance et une vocation spirituelles dont serait porteuse l'attitude philosophique envers le monde, ordonnée à la rationalité, à la vérité et à l'universalité. Le concept culturel détermine l'Europe comme le produit d'une histoire commune forgée par le droit romain, la chrétienté et le rationalisme des Lumières - l'Empire, l'Église et la science concourant à composer l'identité européenne culturelle. L'une et l'autre de ces représentations peinent à rendre compte du triste débouché de cette aventure spirituelle ou culturelle dans le totalitarisme nazi ou stalinien et les pratiques exterminatrices qu'ils ont autorisées. À prendre en revanche au sérieux l'articulation du nihilisme et de l'acosmisme mise en œuvre dans le système européen de la domination totale, on est obligé de poser la question de l'avenir de l'Europe en termes politiques et de dissocier le concept politique de l'Europe de sa prétendue profession spirituelle (la fonction rectrice de la philosophie au service d'une humanité rationnelle) et de sa supposée vocation culturelle (la sécularisation du christianisme dans des institutions juridico-politiques universelles), l'une et l'autre sérieusement dénoncées par l'histoire.

Ce passage d'une Europe philosophique à une communauté politique européenne est inséparable non seulement d'une compréhension critique de la "philosophie politique» mais aussi des blocages de la tradition phénoménologique, husserlienne, heideggérienne et existentialiste à l'égard du politique. On pourrait dire, d'ailleurs, qu'au sein de la phénoménologie au sens strict, seul Jan Patočka a su réactiver la leçon husserlienne et l'infléchir au service d'une élucidation de ce qu'il nommera la post-Europe, proposant une philosophie hérétique de l'histoire qui pense à la fois l'échec historique de l'Europe prise dans l'engrenage de la surcivilisation technique - d'où procède ce qu'on nomme aujourd'hui la globalisation -, et son héritage spirituel, le soin de l'âme qu'il nous invite à penser à partir du platonisme négatif. La reprise patočkienne du diagnostic husserlien parvient à sauver la provenance grecque et philosophique de l'Europe contre son développement culturel et politique en dissociant le soin de l'âme, héritage platonicien, de l'Empire romain et de son legs politique. Cette question du legs philosophique sépare cependant Arendt et Patočka comme elle éloigne Arendt de toute la tradition phénoménologique. Nul testament, fût-il celui d'un platonisme négatif, ne saurait nous faire croire, aux yeux d'Arendt, que l'Europe est encore animée d'une mission philosophique qu'on pourrait préserver et relever de l'histoire politique qui l'a conduite

19. L'analyse distinctive de ces trois concepts d'Europe, philosophique, culturel et politique, a été développée dans É. Tassin, «L'Europe entre philosophie et politique», in L'identité philosophique européenne, J. Poulain et P. Vermeren (dir.), Paris, L’Harmattan, 1993. 
à la catastrophe. S'il nous faut nous situer au plan politique, alors il nous faut en assumer toute la charge: la communauté politique européenne qui se construit politiquement sur les décombres d'une gloire spirituelle passée et encore hantée de prétentions culturelles globalisantes, ne saurait se réclamer d'aucune tradition culturelle (la chrétienté appuyée sur l'Empire) ni se justifier d'aucun testament spirituel (le télos de la vérité, le soin de l'âme...). Elle ne saurait avoir d'autre assise que l'agir en commun des peuples et de leurs représentants, à supposer qu'ils soient capables d'inventer une concitoyenneté effective et de se doter des institutions démocratiques qui rendent effective une politique commune.

Penser l'Europe politiquement et non philosophiquement ou culturellement, ce sera donc, en termes arendtiens, la penser depuis l'action puisque l'agir est le mode d'être politique par excellence. Concrètement, l'idée de l'Europe que perçoit Arendt au sortir de la guerre est enracinée dans l'action des hommes qui se sont battus contre le nazisme, c'est celle des mouvements unifiés de la Résistance européenne. Et pour être conséquent, il faut ajouter immédiatement que le berceau de cette Europe à venir née de l'action concrète des hommes en lutte contre le nazisme ne s'est pas trouvé à Londres mais dans les maquis de la Résistance européenne, en Hollande, en Pologne, en Italie, en France, en Yougoslavie, etc ${ }^{20}$. En 1952, Arendt salue en Henri Frenay, dont elle fait la connaissance par l'intermédiaire de Camus, non seulement le résistant antifasciste, fondateur du réseau Combat, mais aussi le fondateur de la Résistance européenne et le coauteur du Manifeste de Genève de juillet 1944, le militant du Mouvement pour l'Europe et l'homme du congrès de La Haye (1948) d'où est né le Conseil de l'Europe ${ }^{21}$. Et elle réaffirme que seule la perspective d'une fédération

20. Il est remarquable que, de retour de Genève où il avait participé aux premières Rencontres internationales sur l'esprit européen en septembre 1946, Karl Jaspers écrive à Hannah Arendt, le 18 septembre 1946: «J'ai été très impressionné par quelques jeunes hommes de la Résistance française: simples, l’esprit ouvert, sérieux, modestes. On pouvait croire en eux [...]. Il y avait cette chose simple: leur humanité et leur volonté de risquer leur vie pour ça - tout naturellement». Hannah Arendt lui répond le 11 novembre: «Ce que vous écrivez sur les Français de la Résistance m’a fait grand plaisir. Oui, je sais, il y a là encore de vrais hommes; ils sont naturellement une minorité en voie de disparition, mais ils sont néanmoins là et ils sont, ce qui est essentiel, toujours prêts à se battre et à risquer leur vie " (Correspondance. Hannah Arendt - Karl Jaspers, 1926-1969, E. Kaufholz-Messmer (trad. fr.), Paris, Payot, 1996, p. 103 et 114). Le texte de la conférence de K. Jaspers est republié dans Bilan et perspectives, H. Naef et J. Hersh (trad. fr.), Paris, Desclée de Brouwer, 1956, sous le titre: «L'esprit européen», p. 25-60.

21. H. Arendt, lettre à Heinrich Blücher, $1^{\text {er }}$ mai 1952, Correspondance. Hannah Arendt - Heinrich Blücher, 1936-1968, A.-S. Astrup (trad. fr.), Paris, Calmann-Lévy, 1999, p. 233. Il faut toutefois noter qu'après une évocation élogieuse de Frenay, Arendt ajoute cependant qu'il «ferait bien de vraiment faire de la politique au lieu de perdre son temps dans cette crémerie 
européenne offre le moyen de sortir des impasses de l'État-nation. Cette vision d'une Europe fédérale, seule issue d'avenir à la guerre contre le nazisme et au système des États-nations qui y a conduit, s'était imposée à elle avec évidence presque dix ans plus tôt au vu des témoignages de la Résistance européenne.

\section{L'Europe fédérale des Résistances européennes}

Dès 1944, Arendt s'attache à différencier le soi-disant «problème allemand » sur lequel se fixent les interprétations nationalistes de la guerre, du véritable problème que constitue l'orientation à venir de l'Europe mise en cause par le nazisme. Dans l'article qu'elle publie dans la première livraison de Partisan Review au début de l'année 1945, "Approches du "problème allemand" ", elle souligne que l'identification du fascisme ${ }^{22}$ avec le caractère national allemand et son histoire laisse croire que la victoire sur l'Allemagne correspondrait à l'éradication du fascisme alors qu'elle pourrait bien ne faire qu'occulter la «crise européenne ${ }^{23}$. Or le nazisme a crû sur «le vide qui résulte de l'effondrement quasi simultané des structures sociales et politiques de l'Europe». Et «si les mouvements de résistance européens s'opposent si violemment à la restauration de l'ordre ancien, c'est justement parce qu'ils savent qu'elle ne ferait que recréer le même vide ${ }^{24}$. Deux lignes d'interprétations se dessinent et s'opposent ainsi: l'une, attachée aux valeurs d'une Europe des nations d'avant la guerre et qui vise à restaurer l'équilibre des forces antérieur; l'autre qui aura perçu dans la guerre son véritable enjeu européen et qui se doit donc de travailler à une sortie européenne de la guerre. À une interprétation encore nationale du nazisme, Arendt oppose une interprétation politique puisée à la leçon qu'en ont tirée les mouvements de résistance européens.

Les nazis avaient fait de l'Europe l'enjeu de cette guerre. La Résistance européenne a très tôt pris cet enjeu au sérieux: c'est bien le sort de l'Europe qui est en question. Bien avant l'appel «à tous les hommes de la Résistance européenne» lancé par Henri Frenay lors du premier congrès général du mouvement Combat à Alger en mars 1944, et l'affirmation, portée par le projet de déclaration des Résistances européennes de Genève en juillet de la même année, que le moment est venu de «dépasser le dogme de la

de l'Europe qui de toute façon est perdue». Cf. aussi les remarques d'E. Young-Bruehl, Hannah Arendt, J. Roman et É. Tassin (trad. fr.), Paris, Calmann-Lévy, 1999, p. 366-367.

22. Il est d'usage à cette époque aux États-Unis de nommer fascisme l'ensemble des dictatures européennes à commencer par le nazisme.

23. H. Arendt, "Approches du "problème allemand" ", p. 37.

24. Ibid., p. 41. 
souveraineté absolue des États en s'intégrant dans une unique organisation fédérale ", les différents réseaux de résistance européens avaient déjà manifesté l'orientation démocratique, ou socialiste, et fédéraliste de l'Europe à venir au nom de laquelle ils combattaient ${ }^{25}$. À titre d'exemple parmi tant d'autres, le premier numéro de Libérer et fédérer (12 juillet 1942), organe du Mouvement révolutionnaire pour la libération et la reconstruction de la France, exprime ainsi les exigences d'une Fédération européenne: «Le gouvernement révolutionnaire devra préparer, en collaboration avec le gouvernement des autres pays libérés du fascisme et du nazisme, les bases d'une fédération européenne fondée sur la liberté, la paix et la prospérité ${ }^{26}$. La revue Combat affirme, dans son numéro 94 (septembre 1942) que

les États-Unis d'Europe seront bientôt une réalité vivante pour laquelle nous combattons. À la place d'une Europe désunie et asservie à l'Allemagne, ivre de pouvoir, nous formerons avec les autres peuples une Europe unie, organisée sur des bases légales dans un esprit de liberté, d'égalité, de fraternitée ${ }^{7}$.

Ou encore, le premier numéro du journal du Movimento federalista europeo explique en mai 1943 que «l'Union fédérale européenne est la seule formule qui permettrait à l'Europe d'entrer dans un ordre juridique capable d'assurer la coopération pacifique de tous les pays du monde $»^{28}$. Le travail clandestin des internés de Ventotene, entrepris dès juin 1941, avait accompli son œuvre: en France, en Italie, en Belgique, en Pologne, en Hollande, en Norvège, en Tchécoslovaquie, en Yougoslavie, la presse clandestine diffuse cette idée et la résistance s'organise au niveau européen ${ }^{29}$.

Arendt est avertie des positions défendues par ces différents mouvements de Résistance européens, dont la revue londonienne Socialist Commentary reproduit des passages en février 1944. Elle écrira plus tard qu'après «la vaine activité déployée par Briand à la Société des Nations,

25. On trouve une présentation des documents des différents mouvements de résistance européens dans la publication du Centre d'action pour la fédération européenne intitulée L'Europe de demain (Neuchâtel, Éditions de La Baconnière, 1945/ Paris, O. Zeluck, 1945). L'appel de Combat est présenté p. 67.

26. L'Europe de demain, p. 10o. Arendt fait allusion à Libérer et fédérer en disant que c'est là «le principe cardinal de la Résistance française»; et elle ajoute: «et par fédération, on entendait la structure fédérale d'une Quatrième République [par opposition à «l'État centralisé qui est condamné à devenir totalitaire»] s'intégrant à une Fédération européenne».

27. Ibid., p. 93 .

28. Ibid., p. 84 .

29. Assignés à résidence sur l'île de Ventotene en Italie, les militants antifascistes Ernesto Rossi et Altiero Spinelli rédigent un manifeste en faveur d'une Europe libre et d'un Mouvement fédéraliste européen qui circulera clandestinement en Europe. Cf. L'Europe de demain, p. 83. On trouve le texte complet du Manifeste sur le site european navigator (ena): http://www. ena.lu/. 
Hitler déclencha la guerre en promettant de liquider le système désuet de l'État-nation européen et de bâtir une Europe unie ${ }^{30}$. Assumant le déclin de l'État-nation et le relevant dans l'impérialisme, assumant l'épuisement du nationalisme et le relevant sous forme exacerbée dans le nationalisme aryen, Hitler prétend étendre le Reich aux dimensions de l'Europe et faire de celle-ci une Europe aryenne. L'alternative à venir est donc claire: elle n'est plus entre l'Allemagne ou les nations alliées restaurées dans leur ancienne puissance, mais entre l'Europe du troisième Reich ou une Europe fédérée qui aura tiré la leçon de l'effondrement des États-nations et de sa relève nationale-socialiste. L'alternative du fascisme (appuyé sur la liquidation de l'État national et soutenu par une structure impériale) et de la démocratie (accusant les impasses de la structure statonationale et les surmontant par une organisation fédérale de l'Europe) supplante celle des antagonismes nationaux.

L'analyse arendtienne revêt deux aspects: elle indique d'une part en quoi les nations européennes ont failli; elle suggère d'autre part l'issue fédérale de cette crise. La faillite est elle-même double: c'est celle de la structure de classes de la société européenne qui s'est mise à produire des déclassés en nombre, singulièrement en raison du chômage; c'est celle de l'État-nation, l'État national, symbole de la souveraineté du peuple, ne représentant plus le peuple. À ces vérités que sont l'effondrement de la société de classes et la perte de légitimité de l'État-nation, les nazis ont répondu par un double mensonge: celui de la Volksgemeinschaft supposée surmonter l'effritement de la société bourgeoise; celui du «nouvel ordre» européen qui, sous prétexte de rétablir la souveraineté populaire perdue, dégrade en réalité les peuples en races. Outre leur courage propre, la grandeur des mouvements de résistance européens est d'avoir perçu cette compromission du nationalisme dans le fascisme en reconnaissant cette double faillite politique de l'Europe: celle de l'État national, celle du nationalisme lui-même. Aussi leur ennemi est-il le nazisme, pas l'Allemagne.

Ceux qui se levèrent pour faire la guerre combattaient le fascisme, et rien d'autre [...] tous ces mouvements trouvèrent un mot d'ordre politique positif,

30. H. Arendt, «L'Europe et l'Amérique» [1954], Penser l'événement, p. 186. Dans son article de 1944 sur le problème allemand, Arendt cite des passages des journaux clandestins de la résistance hollandaise et polonaise, qu'on trouve reproduits dans la publication du Centre d'action pour la fédération européenne, L'Europe de demain, extraits de Socialist Commentary. En l'absence de certitudes sur la manière dont Arendt a pu constituer son information sur la Résistance européenne, on peut ainsi faire l'hypothèse (qui n'est qu'une possibilité parmi d'autres) qu'elle a eu accès à cette livraison de la revue anglaise ou au moins au matériau recueilli par le Centre d'action pour la fédération européenne. 
indiquant clairement que la nouvelle lutte, quoique très populaire, avait un caractère non national. Ce mot d'ordre, c'était simplement: EUROPE ${ }^{31}$.

Les textes de la Résistance européenne hollandaise, ceux de la Résistance européenne polonaise, ceux de la Résistance européenne française témoignent d'une même analyse: la "crise européenne est avant tout une crise de l'État national $»^{32}$.

Si la guerre est comprise comme une guerre des nations, l'issue militaire de la guerre est la victoire des nations alliées contre l'Allemagne. $\mathrm{Si}$ la guerre est comprise depuis son motif politique et non national, alors elle requiert une issue politique. L'issue politique de la guerre, la véritable sortie européenne d'une guerre dont l'enjeu aura été la configuration de l'Europe, sera donc une sortie de ce qui a donné naissance au nazisme: la crise de l'État national, par une autre configuration de l'Europe: une confédération européenne. Cette orientation fédéraliste de la Résistance européenne est politiquement décisive:

c'est en termes presque identiques que les journaux clandestins français, tchèques, italiens, norvégiens, hollandais, insistent sur ce point comme condition primordiale d'une paix durable - même si, pour autant que je sache, seule la clandestinité française est allée jusqu'à affirmer qu'une structure fédérative européenne doit se fonder sur des structures pareillement fédérales au niveau des États qui la constitueraient [écrit Arendt, épousant entièrement l'analyse de la Résistance européenne] ${ }^{33}$.

Une réponse «nationale» au nazisme serait encore une victoire du nazisme qui est l'exacerbation du nationalisme et de l'impérialisme de la race ${ }^{34}$.

De ce point de vue, il n'est pas étonnant, d'une part, qu'Arendt souligne tout ce qui sépare la conception gaullienne de la guerre de celle des résistants de l'intérieur. De Gaulle raisonne encore selon une orientation nationale; il pense que le problème allemand est le cour de cette guerre et que de lui dépend son issue, exigeant ainsi l'annexion de la Rhénanie puis, après la libération de Paris, sa simple occupation ${ }^{35}$. Arendt lui oppose

31. H. Arendt, "Approches du "problème allemand" ", p. 42.

32. Ibid., p. 43.

33. On relèvera la proximité de cette dernière réflexion avec les observations réunies dans L'Europe de demain, qui laisse penser qu'à défaut d'avoir lu cet ouvrage (dont l'achevé d'imprimer est de mars 1945) à l'époque où elle rédige son article, Arendt en connaît le matériau qu'il recueille.

34. Rédigée en 1944, cette analyse s'appuie encore sur la notion d'impérialisme de race et non sur le concept de totalitarisme dont la formulation achevée n'est acquise qu'en 1948-1949.

35. De Gaulle est à ses yeux un «rappel des forces d'avant-hier [...], le seul à représenter vraiment le patriotisme et le nationalisme au sens d'autrefois [...], le seul homme d'État 
Georges Bidault, successeur de Jean Moulin à partir de juin 1943 comme président du Conseil national de la résistance qui, devenu ministre des Affaires étrangères, s'adresse aux prisonniers allemands en leur souhaitant de «trouver place bientôt dans une Allemagne libre et une Europe libre ${ }^{36}$. Il n'est pas indifférent, d'autre part, qu'elle souligne aussitôt que le retour des gouvernements en exils correspond à une restauration des structures nationales, lesquelles font rapidement taire «ce nouveau sentiment de solidarité européenne ${ }^{37}$. De même qu'elle sera plus tard sensible aux mots de René Char déplorant qu'avec le temps de la Résistance cesse celui d'une expérience proprement politique au profit d'une restauration des appareils de gouvernements ${ }^{38}$, Arendt note dès 1944 que cette restauration s'emploie à affaiblir et disperser les mouvements de résistance et à détruire la renaissance des peuples européens qui s'était manifestée à travers elle. La restauration que visent les gouvernements en exil à leur retour au pouvoir repose en effet sur les trois concepts fondamentaux - sécurité collective, sphère d'intérêt, alliances nationales - qui ont joué un rôle décisif dans la préparation de la guerre et son déclenchement. «Il n'y a rien à attendre de la restauration ", conclut Arendt puisqu'elle est aussi la restauration de ce qui a conduit à la Seconde Guerre mondiale et au "problème allemand». À rebours de celle-ci, il ne saurait donc y avoir d'autre alternative pour préserver la paix et la liberté que «la voie choisie par la résistance européenne» 39 .

Certes, la voie fédérale ne sera pas celle que l'Europe adoptera au lendemain de la Seconde Guerre. La déclaration des Résistances européennes restera lettre morte, le congrès de La Haye devra reconnaitre que la voie fédéraliste est impuissante à surmonter le retour en force de la souveraineté nationale qui se réaffirme aussitôt, comme si la guerre n'avait rien appris aux gouvernants mais seulement aux combattants. Et il faudra toute la ruse machiavélienne des artisans de la Communauté du charbon et de l'acier puis, mais sans succès, de la Communauté européenne de défense, pour substituer à la construction d'une Europe fédérale, qu'Arendt a toujours soutenue mais que les gouvernements de restauration combattront, une intégration fonctionnelle économique dont le bénéfice politique mettra

national qui reste en Europe, pour qui le "problème allemand est le centre de l'univers", pour qui "la guerre est réellement un conflit national et non pas idéologique" » (H. Arendt, "Approches du "problème allemand" ", p. 49-50).

36. Ibid., p. 43.

37. Ibid., p. 48.

38. R. Char, «Feuillets d'Hypnos», $\mathrm{n}^{\circ} 195$ (que cite Arendt, CC, préface, p. 12) et n 220, p. 137 et 144 .

39. H. Arendt, "Approches du "problème allemand" », p. 51. 
près de cinquante ans à se manifester. La fédéralisation de l'Allemagne, qui devait préparer «le peuple allemand à la fédération européenne dont on espère l'avènement» écrit-elle, en 1950 ne sera pas beaucoup plus efficace ${ }^{40}$. Et pourtant, Arendt maintiendra que, comme tous les autres problèmes européens, la question des conditions de vie en Allemagne «ne pourrait se résoudre qu'au sein d'une Europe fédérée ${ }^{41}$. Quatre ans plus tard, et donc dix ans après l'éloge appuyé de la Résistance européenne, revenant sur les rapports de "L'Europe et [de] l'Amérique» (1954), elle souligne le risque que l'image que l'Europe se fait de l'Amérique puisse marquer «le début d'un nouveau nationalisme paneuropéen ». Mais elle ajoute cependant: «Peut-être est-ce un optimisme injustifié qui nous fait espérer que l'émergence d'une Europe fédérée et la dissolution du système actuel de l'État-nation feront du nationalisme une relique du passé " ${ }^{42}$. Car l'enjeu est encore clair : tenir ferme la frontière qui sépare l'européanisme antiaméricain « des efforts tout à fait nécessaires et salubres pour fédérer les nations européennes » ${ }^{43}$. La fédération de l'Europe est en effet «très probablement une condition sine qua non de sa survie » ${ }^{44}$. Aussi l'article de 1954 peut-il affirmer en conclusion qu'aux «craintes justifiées que l'Europe éprouve quant à son identité spirituelle» et que la restauration des gouvernements nationaux n'a bien évidemment pas levées, s'ajoutent aussi, dix ans après la fin de la guerre, «les appréhensions plus vives encore qu'elle nourrit quant à sa simple survie matérielle ${ }^{45}$. Devant la restauration du nationalisme souverain, que ne nuancent guère la signature du futur traité de Rome et la formation de la CEE, Arendt ne reviendra pas sur la question européenne jusqu'à sa mort en 1975 .

La version arendtienne de la crise de l'humanité européenne consiste ainsi dans une analyse politique de la conjonction entre l'État national, incarnation de la souveraineté populaire et structure de gouvernement, et le nationalisme, idéologie destinée à justifier la fiction d'une coïncidence entre le peuple et la nation dans une commune souveraineté ${ }^{46}$. Le nazisme porte cette conjonction à son plus haut point d'incandescence meurtrière en déployant un système de domination totale qui, substituant l'aryanité

40. H. Arendt, «Après le nazisme: les conséquences de la domination» [1950], Penser l'événement, p. 74 .

41. Ibid., p. 77.

42. H. Arendt, «L'Europe et l'Amérique» [1954], p. 185.

43. Ibid., p. 186.

44. Ibid., p. 195.

45. Ibid., p. 196.

46. Je me permets de renvoyer, pour l'analyse de cette fiction, à É. Tassin, «Le peuple ne veut pas", in Hannah Arendt. Crises de l'État-nation, A. Kupiec et al. (éd.), Paris, Sens \& Tonka, 2007, p. 301-315. 
à la nation et le Reich à l'État-nation, vise à soumettre l'Europe unifiée à l'impérialisme de race. Agissant de concert, avec la lucidité que procure l'action commune et qui fait encore défaut aux gouvernements en exil soumis aux tractations diplomatiques, les combattants de la Résistance européenne, reconnaissant les causes et l'enjeu de cette guerre, comprirent qu'il revenait aux Européens d'opposer une autre Europe unie, démocratique et post-nationale, à celle que leur promettait le totalitarisme. La seule issue politique à la guerre consiste alors en l'invention d'une fédération ou confédération européenne mettant fin aux mythes conjoints du peuple souverain, du peuple incarné dans la nation, du pouvoir d'État compris comme domination. Le fédéralisme européen est, comme l'a écrit JeanClaude Poizat, "l'invention d'une politique non souveraine, seul espoir pour l'Europe ${ }^{47}$ qui aura surmonté le traumatisme de la guerre, assumé la rupture de la tradition et relevé sa négation nazie. C’est alors, mais à partir du plan politique, que pourront éventuellement être reposées les questions de l'esprit européen, de la culture européenne, de l'identité européenne, voire de ce qu'il en est de la prétendue vocation européenne à la philosophie - questions que seul, il faut le noter, au cours des trente années qui suivirent, Jan Patočka aura à cœur de reprendre depuis «l'autre Europe», celle de l'Est, avant que d'autres à l'Ouest y reviennent aussi, à commencer par Jacques Derrida. Mais, du point de vue d'Arendt, ces interrogations sur l'Europe spirituelle ou culturelle ne sauraient être posées que sous condition de ne pas livrer l'Europe politique à une intelligence philosophique de surplomb supposée indépendante de l'histoire, et de ne pas subordonner le plan politique aux plans spirituel, culturel ou communautaire comme si l'union politique européenne dépendait pour se déployer d'autre chose que d'un agir ensemble concitoyen. Il n'est pas sûr aujourd'hui que cette leçon arendtienne ait encore été vraiment entendue.

Étienne TAssin

47. Sur le fédéralisme européen, voir l'analyse judicieuse de J.-C. Poizat, «L'invention d'une politique non souveraine: Arendt et l'espoir européen", in Hannah Arendt. Crises de l'État-nation, p. 245-261. 